\title{
Efecto antinociceptivo y sobre la neuroconducta de la amitriptilina en dosis escalonada en roedores
}

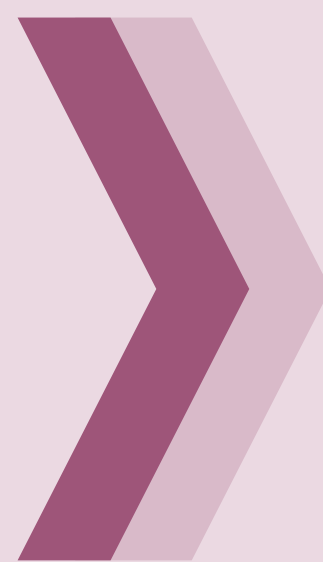

\author{
Amitriptyline effect on the \\ antinociception and the \\ neurobehavior in escalation \\ dose in rodents
}

\author{
Alberto Alcibiades Salazar-Granara, 1,2,3,4a \\ Cynthia Evelyn Arizanca-Callata, ${ }^{1,2, b}$ Escarlet Inés Cerón-Uribe, ${ }^{1,2, b}$ \\ Jesús Rafael Custodio-Aguilar, ${ }^{1,2, b}$ \\ Amanda Liset Hernández-Luján,1,2,b \\ Luis Munayco-Castro, ${ }^{1,2, b}$ Juan Carlos Pante-Medina ${ }^{2,3, c}$
}

1 Facultad de Medicina Humana de la Universidad San Martín de Porres (FMH-USMP).

2 Centro de Investigación de Medicina Tradicional y Farmacología de la FMH-USMP.

3 Bioterio de la FMH-USMP.

a Médico; doctor en Medicina.

b Estudiante de Medicina Humana.

c Técnico especialista en investigación biomédica.

\section{RESUMEN}

Objetivo. Evaluar el efecto antinociceptivo agudo y sobre la neuroconducta de la amitriptilina en dosis escalonadas. Materiales y métodos. Se utilizaron 82 ratones de aproximadamente $28 \mathrm{~g}$, distribuidos en 10 grupos; se les suministró vía oral: agua destilada $(0,1$ $\mathrm{mL} / 10 \mathrm{~g})$, tramadol $(20 \mathrm{mg} / \mathrm{Kg})$, cafeína (32 $\mathrm{mg} / \mathrm{Kg})$, diazepam (32 mg/Kg)y amitriptilina en dosis escalonada (10-50 mg/Kg). Se emplearon las pruebas de la "placa caliente" y de Irwin. Se aplicaron las pruebas estadisticas de ANOVA, Tukey, coeficiente de correlación de Pearson y la exacta de Fisher. Resultados. La amitriptilina incrementó el umbral del dolor según el incremento de la dosis administrada, de 30 a $50 \mathrm{mg} / \mathrm{Kg}(\mathrm{p}<0,05$; IC 95\%). En las mismas dosis, se observó la presencia de sedación en $46,9 \%, 56,2 \%$, y $81,2 \%$, respectivamente. Conclusiones. Se demostró la acción antinociceptiva aguda de la amitriptilina y su relación dosis-respuesta en el rango de 30-50 mg/ $\mathrm{Kg}$, asimismo, se describieron manifestaciones neuroconductuales como: sedación, piloerección, incoordinación motora, salto, respiración anormal y marcha anormal.

Palabras clave: amitriptilina; nocicepción; analgesia

\begin{abstract}
Objective. To evaluate the antinociceptive effect in acute pain and neurobehavioral of amitriptyline in dose response. Materials and methods. It was used 82 mice of approximately $28 \mathrm{~g}$, distributed in 10 groups. It was given by orally: distilled water $(0.1 \mathrm{ml} / 10 \mathrm{~g})$, tramadol (20 mg/Kg), caffeine (32 mg/Kg), diazepam (32 mg/ $\mathrm{Kg}$ ), and amitriptyline, in doses staggered (10 to $50 \mathrm{mg} / \mathrm{Kg}$ ). These were used the hot plate test and the Irwin test. The statistics validation was made trougth the next test: one way ANOVA, Tukey, Pearson correlation coefficient and the Fisher exact test. Results. Amitriptyline increased the pain threshold by increasing the administered dose of 30 to $50 \mathrm{mg} / \mathrm{kg}(p<0.05 ; 95 \% \mathrm{Cl})$. At the same doses, it was shown presence of sedation in $46.9,56.2 \%$ and $81.2 \%$, respectively. Conclusions. It was demonstrated the aninociceptive acute effect of amitriptyline. Also, it was shown sedation, piloerection, motor incoordination, jumping, abnormal breathing, and abnormal gait.
\end{abstract}

Key words: amitriptyline; nociception; analgesia 


\section{INTRODUCCIÓN}

La amitriptilina es un antidepresivos tricíclicos (ATC), cuyo mecanismo de acción es inhibir la recaptación de serotonina y norepinefrina en la misma proporción. ${ }^{1}$ Asimismo, presenta efecto antimuscarínico y es antagonista de los receptores histamínicos $\mathrm{H}_{1}$ y adrenérgicos $\alpha_{1}{ }^{2}$ En similitud, los ATC y los inhibidores selectivos de la recaptación de serotonina (IRSS), aumentan el nivel de monoaminas en las interneuronas inhibitorias, excitatorias y de neuronas de proyección, tanto en la presinapsis como en la postsinapsis; en consecuencia, aumentan la inhibición provocada por los receptores gabaérgicos. ${ }^{3}$

Por otra parte, se propone que los ATC ejercen acción analgésica mediante el antagonismo de los canales de calcio en el ámbito presináptico, inhibiendo la liberación de hormonas y neurotransmisores. ${ }^{4}$ En el dolor nociceptivo agudo, el sistema nervioso central (SNC) recibe la señal para reducir el dolor percibido. ${ }^{5}$ En varios estudios en humanos se ha comprobado que la amitriptilina tiene efecto analgésico y es efectiva frente al dolor neuropático. ${ }^{6-9}$ En roedores se ha corroborado el efecto analgésico mediante la prueba de la "placa caliente" (hot plate test). ${ }^{10}$

Según la escala analgésica del dolor, de la Organización Mundial de la Salud (OMS), ${ }_{1}^{11}$ la amitriptilina es considerada como coanalgésico; en tal sentido, en la práctica clínica se usan dosis establecidas para sus aplicaciones principales (antidepresivo):12,13 sin embargo, lo que aún no se ha dilucidado es su potencial eficacia analgésica relacionada con la dosis, especialmente ante el dolor agudo, ${ }^{14}$ como tampoco sus posibles efectos adversos, que son consecuencia de la acción sobre otros sistemas de neurotransmisores, como los de tipo anticolinérgico (sedación, sequedad de boca, estreñimiento, retención urinaria, visión borrosa, midriasis y cicloplejía) y serotoninérgico (náuseas y vómitos, taquicardia e hipotensión postural), entre otros. ${ }^{15}$

Existen modelos experimentales en roedores, que permiten explorar la antinocicepción aguda, como la prueba de la "placa caliente", ${ }^{16,17}$ y la neuroconducta en el roedor, como la prueba de Irwin. ${ }^{18,19}$ Este estudio explora el efecto antinociceptivo de la amitriptilina en dosis escalonada, en un modelo experimental de dolor agudo en ratones, y sus posibles efectos sobre la neuroconducta.

\section{MATERIALES Y MÉTODOS}

Estudio experimental, realizado en el Centro de Investigación de Medicina Tradicional y Farmacología (CIMTFAR), de la Facultad de Medicina Humana de la Universidad de San Martín de Porres (FMH-USMP), durante el periodo diciembre 2013 - noviembre 2014. Se utilizaron 82 ratones albinos machos de la especie Mus musculus de aproximadamente $28 \mathrm{~g}$ de peso corporal, los cuales fueron separados y colocados en jaulas, provistas de alimento balanceado y agua ad libitum. La aclimatación se dio en el bioterio de la FMH-USMP, a una temperatura de $22-25^{\circ} \mathrm{C}$, con humedad inferior a $70 \%$, ciclos de luz/oscuridad de 12 horas y niveles de ruido menores de $70 \mathrm{~dB}$. La distribución y asignación aleatorizada de los grupos experimentales se realizó por sorteo.20,21

Como material de laboratorio se empleó una sonda metálica para la administración perioral, una balanza analítica, jaulas con capacidad para varios ratones adaptada con viruta, depósitos de comida y agua; cubierta con la menor exposición a la luz, cubículos de vidrio translúcido, equipo de "placa caliente" marca Thermo Scientific ${ }^{\mathrm{TM}}$ Cimarec $^{\mathrm{TM}}$ $\left(5-540{ }^{\circ} \mathrm{C}\right)$ y una campana de vidrio trastúcido. Entre el material químico empleado estuvieron el agua destilada, amitriptilina en tabletas de $25 \mathrm{mg}$, cafeína $100 \mathrm{mg} / 20 \mathrm{~mL}$, diazepam ampolla de 10 $\mathrm{mg} / 2 \mathrm{~mL}$ y tramadol $50 \mathrm{mg} / 1 \mathrm{~mL}$.

Se aplicó un sistema de triple ciego para la administración de las sustancias químicas, observación de manifestaciones y análisis estadístico. ${ }^{22}$ Asimismo, se realizó un entrenamiento virtual previo para la evaluación de las manifestaciones de dolor de los ratones, empleando los programas Virtual Pharmacology Lab ${ }^{\odot 23}$ y Microlabs ${ }^{\odot}, 24$ y también un ensayo práctico con un roedor vivo. Para el control de la humedad, el ruido, y la temperatura de la sala de experimentación, se empleó un higrómetro digital Thermo-Hygro ${ }^{\odot}$, marca VWR International ( $\mathrm{T}^{\circ}: 5-34^{\circ} \mathrm{C}$; humedad: $32-82 \%$ ) y un sensor de ruido marca Digital Sound Level Meter ${ }^{\odot}(60-120 \mathrm{~dB})$. 
Para la observación y detección de los efectos adversos de la sustancia estudiada sobre el comportamiento general y para evaluar la neurotoxicidad, se empleó la prueba de Irwin, durante la primera hora de la prueba, en cuatro momentos, con intervalos de 15 minutos; el observador marcaba con un punto de control a los 15, 30, 45 y 60 minutos. Se evaluó la presencia o ausencia de letalidad, convulsiones, cola de Straub, sedación, excitación, marcha anormal (en círculos o en puntas de pie), saltos, incoordinación motora, piloerección, estereotipias (oler, masticar, o movimientos de cabeza), contorsiones abdominales, sacudidas de cabeza, escozor y alteración de la respiración. ${ }^{18}$

En la prueba de la "placa caliente", que consiste en poner al ratón en una placa gruesa de aluminio (10 mm) a $55 \pm 1^{\circ} \mathrm{C}$ y cubrirlo con una campana de vidrio, se registró el tiempo de reacción del roedor expuesto al calor (cuando saltó o lamió su patas), hasta un máximo de 30 segundos, momento en el que se retiraba al roedor. Se realizó una valoración inicial y luego tres mediciones más, a los 60,90 y 120 minutos, por cada ratón. ${ }^{17}$

Se conformaron diez grupos experimentales, con aproximadamente ocho ratones cada uno: al grupo 1 o control, no se le administró ninguna sustancia; grupo 2 o placebo, agua destilada $(0,1$ $\mathrm{mL} / 10 \mathrm{~g})$; grupo 3 de control, tramadol $(20 \mathrm{mg} / \mathrm{Kg})$; grupo 4 de control, cafeína ( $32 \mathrm{mg} / \mathrm{Kg}$ ); grupo 5 de control, diazepam (32 mg/Kg); grupos 6, $7,8,9$ y 10 , recibieron amitriptilina a dosis escalonada entre 10 y $50 \mathrm{mg} / \mathrm{Kg}$. La administración de las sustancias fue por vía oral.

Este estudio contó con la aprobación del Instituto de Investigación de la FMH-USMP. Asimismo, se siguieron los principios éticos de la Declaración de Helsinki y de la Ley General de Salud del Perú (Ley 26842). ${ }^{25,26}$

\begin{tabular}{cccccccccc}
\multicolumn{8}{c}{ Tabla 1. Tiempo de reacción de los roedores expuestos al calor durante la prueba de la } \\
"placa caliente", post administración de amitriptilina en dosis escalonadas.
\end{tabular}

* Prueba ANOVA $(p<0,05)$ y métodos de comparación múltiple de Tukey y Student-Newman-Keuls $(p<0,05)$ para la prueba de la "placa caliente", de los grupos. A los 60 minutos, los grupos 6, 7 y 8 tuvieron mejor respuesta, en comparación con los grupos 1 y 2; a los 90 minutos, el grupo 6 fue mejor que los grupos 1 y 2; a los 120 minutos, grupo 8 fue mejor que el grupo 1, con una diferencia estadísticamente significativa.

En donde: $\mathrm{X}=$ media aritmética; $\mathrm{DE}=$ desviación estándar. 
Los datos obtenidos fueron tabulados y expresados en frecuencias, medias y desviación estándar, según corresponde. Se aplicaron las pruebas de ANOVA (luego de haber aplicado el test de normalidad de Kolmogorov-Smirnov), Fisher y coeficiente de correlación de Pearson. Se estableció un nivel de significancia estadística de $p<0,05$, con un intervalo de confianza de $95 \%$. Se usaron como soporte informático Microsoft Excel Windows $2010^{\circ}$ y el programa GraphPad Prism ${ }^{\odot}$ versión 5.

\section{RESULTADOS}

En la tabla 1 se muestra el tiempo de reacción de los roedores expuestos al calor durante la prueba de la "placa caliente", por cada grupo experimental. Se encontró que el efecto antinociceptivo de la amitriptilina se presentó cuando fue administrada en concentraciones mayores de $30 \mathrm{mg} / \mathrm{Kg}$ y a partir de los 60 minutos de su ingesta. Se encontró una correlación lineal positiva entre la dosis administrada de amitriptilina, de manera escalonada (de 10 a $50 \mathrm{mg} / \mathrm{Kg}$ ), y el efecto (tiempo de reacción de los roedores expuestos al calor) durante la prueba de la "placa caliente" ( $r=0,4909 ; p=0,013$; IC 95\%; coeficiente de determinación $r^{2}=0,2410$, según la prueba de correlación de Pearson) (Gráfico 1).

En la tabla 2 se presentan las manifestaciones neuroconductuales de los roedores, observadas durante la prueba de Irwin, luego de la administración de amitriptilina, a dosis escalonadas (de 10 a 50 $\mathrm{mg} / \mathrm{Kg}$ ). En la evaluación neurofarmacológica mediante la prueba de Irwin, al comparar los grupos experimentales (a quienes se les administró amitriptilina a dosis escalonadas) con los grupos de control, observamos que se presentaron: sedación, en el grupo control y en el grupo $10(p<0,05)$; excitación, en los grupos 6 y $7(p<0,05)$; incoordinación motora, en el grupo $10(p<0,05)$ y respiración anormal, en los grupos 9 y 10 ( $p<0,05)$ (Tabla 3).

En el gráfico 2 se evidencia que la sedación es cada vez mayor según se aumenta la dosis de amitriptilina (de $10-50 \mathrm{mg} / \mathrm{Kg}$ ), con valores de: $18,8 \%, 40,6 \%, 46,9 \%, 56,2 \%$ y $81,2 \%$, respectivamente. Por otra parte, con respecto a la presencia de la incoordinación motora, se observó la misma tendencia: $0 \%, 13 \%, 16 \%, 44 \%$ y $72 \%$ (Gráfico 3). La respiración anormal se presentó según la dosis escalonada de amitriptilina: 0\%, $25 \%, 34,3 \%, 56,2 \%$ y $50 \%$ (Gráfico 4 ).

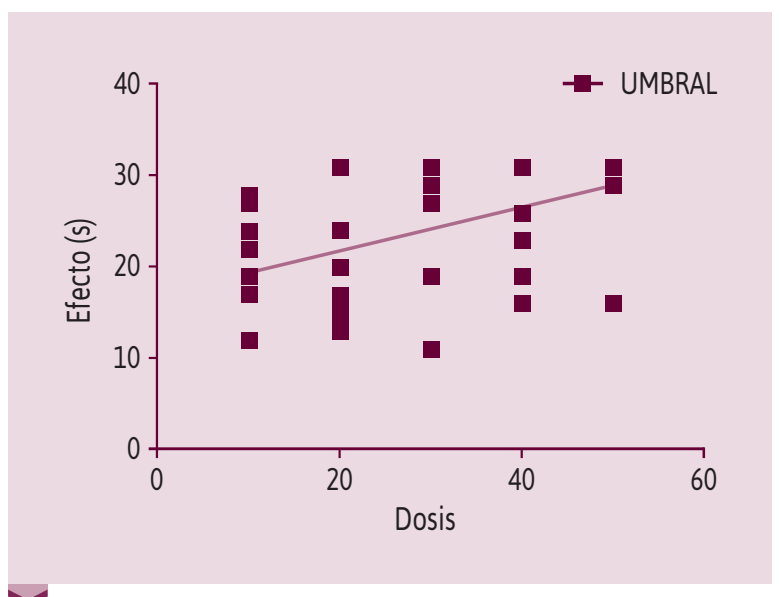

Gráfico 1. Relación dosis-efecto de la amitriptilina en la prueba de la "placa caliente", en los primeros 60 minutos post administración.

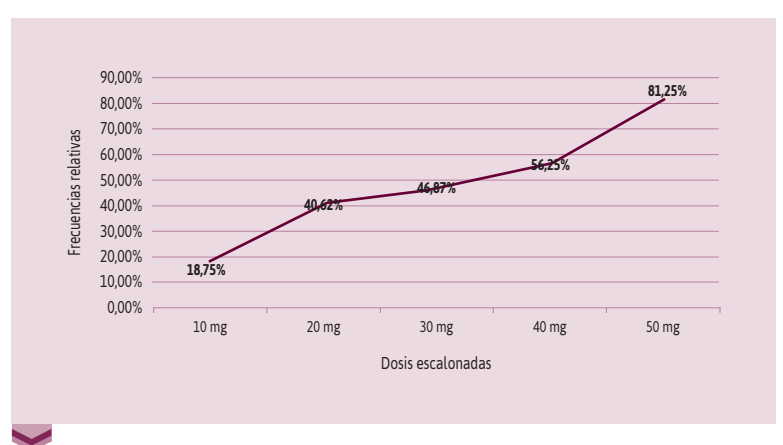

Gráfico 2. Presencia de la sedación en la prueba de Irwin, post administración de amitriptilina a dosis escalonadas.

\section{DISCUSIÓN}

La prueba de la "placa caliente" provoca un dolor somático clásico; es una prueba nociva de calor inducido que ha demostrado proveer un buen índice de la actividad antinociceptiva de las drogas en humanos (actividad analgésica de alguna sustancia). Este ensayo mide el dolor agudo, debido a la activación de nociceptores periféricos por medio de un umbral de nocicepción. ${ }^{17}$

En este estudio se demostró la actividad antinociceptiva de la amitriptilina, administrada en el rango de dosis de 30 a $50 \mathrm{mg} / \mathrm{Kg}$, con una correlación lineal positiva, según la prueba de Pearson. En otra investigación se demostró el mismo efecto antinociceptivo de la amitriptilina en la dosis de $50 \mathrm{mg} / \mathrm{Kg}^{27}$ además, en ese estudio se observó que la amitriptilina administrada en dosis menores de $50 \mathrm{mg} / \mathrm{Kg}$ de amitriptilina produce un efecto antinociceptivo equipotente al 
del tramadol. Según otros autores, en dosis de 50 $\mathrm{mg} / \mathrm{Kg}$, el efecto es superior y equipotente al de la morfina. ${ }^{28}$ Por lo tanto, se plantea la probabilidad de la intermediación de receptores opioides en el efecto antinociceptivo de la amitriptilina, lo cual debería ser explorado en futuros trabajos. ${ }^{29}$

Sin embargo, otras vías también pueden explicar el efecto antinociceptivo de la amitriptilina, entre ellas la regulación del calcio, puesto que esta droga es antagonista de los canales de calcio dependiente de voltaje a nivel presináptico, inhibiendo la liberación de hormonas y neurotransmisores.,30 Asimismo, actúa inhibiendo la recaptación de monoaminas (aumentando su concentración en la sinapsis), que participan en las proyecciones entre el tronco cerebral y los núcleos de las astas dorsales de la médula espinal, implicadas en la modulación nociceptiva, potenciando los sistemas descendentes inhibidores de la respuesta dolorosa. ${ }^{31,32}$ También posee otras acciones, tales como como bloqueador de receptores muscarínicos colinérgicos, histamínicos $\mathrm{H}_{1}$ y adrenérgicos que participan en

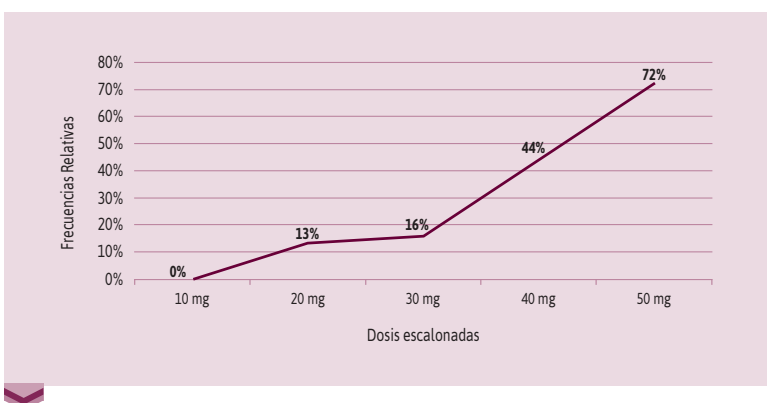

Gráfico 3. Presencia de la incoordinación motora en la prueba de Irwin, post administración de amitriptilina a dosis escalonadas.

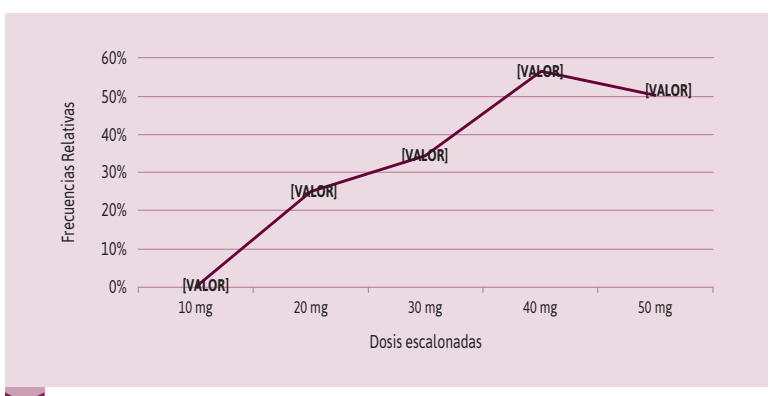

Gráfico 4. Presencia de la respiración anormal en la prueba de Irwin, post administración de amitriptilina a dosis escalonadas.

\begin{tabular}{|c|c|c|c|c|c|c|c|c|}
\hline Variables & $\begin{array}{l}\text { Grupo } \\
\text { control }\end{array}$ & $\begin{array}{l}\text { Cafeína } \\
\text { Grupo } 4\end{array}$ & $\begin{array}{l}\text { Diazepam } \\
\text { Grupo } 5\end{array}$ & $\begin{array}{c}\text { Amitriptilina } \\
10 \mathrm{mg} / \mathrm{Kg} \\
\text { Grupo } 6\end{array}$ & $\begin{array}{c}\text { Amitriptilina } \\
20 \mathrm{mg} / \mathrm{Kg} \\
\text { Grupo7 }\end{array}$ & $\begin{array}{c}\text { Amitriptilina } \\
30 \mathrm{mg} / \mathrm{Kg} \\
\text { Grupo } 8\end{array}$ & $\begin{array}{c}\text { Amitriptilina } \\
\text { 40mg/Kg } \\
\text { Grupo } 9\end{array}$ & $\begin{array}{c}\text { Amitriptilina } \\
50 \mathrm{mg} / \mathrm{Kg} \\
\text { Grupol0 }\end{array}$ \\
\hline Sedación & $\mathrm{Si}^{*}$ & Sí & No $\ddagger$ & Sił & Sí & Sí & Sí & $\mathrm{Si}^{*}$ \\
\hline Excitación & $\mathrm{Si}^{*}$ & Sí & No & $\mathrm{Si}^{*}$ & $\mathrm{Si}^{*}{ }^{*}$ & Sí & $\mathrm{No}^{*}$ & $\mathrm{No}^{*}$ \\
\hline Piloerección & Sí & No & Sí & Sí & Sí & Sí & Si & $\mathrm{Si}$ \\
\hline Incord. motora & $\mathrm{No}^{*}$ & Not & No & No & Sí & Sí & $\mathrm{Si}$ & $\mathrm{Si}^{\dagger}{ }^{*}$ \\
\hline Salto & No & No & No & Sí & Sí & Sí & No & No \\
\hline Respiración anormal & $\mathrm{No}^{*}$ & No $\dagger$ & Síł & Sił & Sił & Sí & $\mathrm{Si}^{*}$ & $\mathrm{Si}^{*}{ }^{\star}$ \\
\hline Marcha anormal & No & No & Sí & No & Sí & Sí & Si & No \\
\hline
\end{tabular}

* Prueba exacta de Fisher $(p<0,05)$ para la prueba de Irwin. Comparación entre: grupo de control y grupo de amitriptilina $50 \mathrm{mg} / \mathrm{Kg}$, grupo de control y grupos de amitriptilina 10, 20, 40 y $50 \mathrm{mg} / \mathrm{Kg}$, grupo de control y grupo de amitriptilina $50 \mathrm{mg} / \mathrm{Kg}$, grupo de control y grupo de amitriptilina 40 y $50 \mathrm{mg} / \mathrm{Kg}$.

$\dagger$ Prueba exacta de Fisher $(p<0,05)$ para la prueba de Irwin. Comparación entre: grupo de cafeína y grupos de amitriptilina 30-50 mg/Kg, grupo de cafeína y grupo de amitriptilina 20 y $40 \mathrm{mg} / \mathrm{Kg}$, grupo de cafeína y grupo de amitriptilina $50 \mathrm{mg} / \mathrm{Kg}$; grupo de cafeína y grupo de amitriptilina 40 y $50 \mathrm{mg} / \mathrm{Kg}$.

\# Prueba exacta de Fisher $(p<0,05)$ para la prueba de Irwin. Comparación entre: grupo de diazepam y grupo de amitriptilina $10 \mathrm{mg} / \mathrm{Kg}$; grupo de diazepam y grupo de amitriptilina 10 y $20 \mathrm{mg} / \mathrm{Kg}$. 


\section{Anta Méálica Pernana}

\begin{tabular}{|c|c|c|c|c|c|c|c|c|c|c|}
\hline \multirow{2}{*}{ Variable } & \multicolumn{2}{|c|}{$\begin{array}{l}\text { Amitriptilina } \\
10 \mathrm{mg} / \mathrm{Kg}\end{array}$} & \multicolumn{2}{|c|}{$\begin{array}{l}\text { Amitriptilina } \\
20 \mathrm{mg} / \mathrm{Kg}\end{array}$} & \multicolumn{2}{|c|}{$\begin{array}{c}\text { Amitriptilina } \\
30 \mathrm{mg} / \mathrm{Kg}\end{array}$} & \multicolumn{2}{|c|}{$\begin{array}{l}\text { Amitriptilina } \\
40 \mathrm{mg} / \mathrm{Kg}\end{array}$} & \multicolumn{2}{|c|}{$\begin{array}{c}\text { Amitriptilina } \\
50 \mathrm{mg} / \mathrm{Kg}\end{array}$} \\
\hline & $n$ & $(\%)$ & $n$ & $(\%)$ & $n$ & $(\%)$ & $n$ & $(\%)$ & $n$ & $(\%)$ \\
\hline Sedación & 6 & 18,8 & 13 & 40,6 & 15 & 46,9 & 18 & 56,2 & 26 & 81,2 \\
\hline Excitación & 5 & 16 & 2 & 6 & 9 & 28 & 1 & 3 & 2 & 6 \\
\hline Piloerección & 14 & 44 & 15 & 46 & 10 & 31 & 13 & 40 & 14 & 43 \\
\hline $\begin{array}{l}\text { Incoordinación } \\
\text { motora }\end{array}$ & 0 & 0 & 4 & 13 & 5 & 16 & 14 & 44 & 23 & 72 \\
\hline Salto & 7 & 21,9 & 1 & 3,1 & 11 & 34,4 & 0 & 0 & 1 & 3,1 \\
\hline $\begin{array}{l}\text { Respiración } \\
\text { anormal }\end{array}$ & 0 & 0 & 8 & 25 & 11 & 34,3 & 18 & 56,2 & 16 & 50 \\
\hline Marcha anormal & 1 & 3 & 11 & 34 & 9 & 28 & 2 & 6 & 0 & 0 \\
\hline
\end{tabular}

la modulación de la respuesta nociceptiva. ${ }^{33,34}$ Asimismo, se reconoce que la amitriptilina es antagonista de los receptores histaminérgicos $\mathrm{H}_{1}$, que intervienen en la percepción de dolor. ${ }^{35}$

Por otro lado, en este estudio, la prueba de Irwin reveló que la amitriptilina indujo la sedación, la piloerección, la incoordinación motora, el salto, la respiración anormal (aumento-disminución) y la marcha anormal. El efecto de sedación se presentó con mayor frecuencia cuando fue administrada en dosis de 40 y $50 \mathrm{mg} / \mathrm{Kg}$. Esto se debería a la actividad antagonista de la amitriptilina sobre los receptores $\mathrm{H}_{1}$ y adrenérgicos ${ }_{1}$ en el cerebro. ${ }^{36}$

Considerando que a dosis de $50 \mathrm{mg} / \mathrm{Kg}$ se presentó el efecto antinociceptivo más eficaz, esta característica farmacodinámica podría ser una ventaja, para el uso de esta droga en eventos de dolor agudo, pues el alivio del dolor también se puede lograr tranquilizando al paciente. ${ }^{37}$

La incoordinación motora es un efecto extrapiramidal reconocido de la amitriptilina, debido a su acción sobre los receptores muscarínicos, lo cual se manifiesta en el paciente mediante la alteración de la postura, tono muscular, equilibrio y coordinación. ${ }^{38}$ La piloerección es una manifestación que se relaciona con la estimulación de los receptores ${ }_{1}$ adrenérgicos, evento que se explica por el mecanismo de la amitriptilina de inhibición de la recaptación de monoaminas. ${ }^{39}$

Los saltos en el roedor pueden ser la expresión de neuroexcitación, que se observó en dosis bajas de amitriptilina; los mecanismos implicados pueden estar mediados por vías adrenérgicas y serotoninérgicas. ${ }^{40}$ Es preciso resaltar que las principales limitaciones que presenta este estudio radican en la dificultad para la exploración exacta de los niveles y mecanismos de acción; por ello, se recomienda que esto se indague y considere en futuros estudios. Sin embargo, considerando que la amitriptilina es una droga empleada en la clínica, este estudio brinda información útil para la comunidad médica.

\section{CONCLUSIONES}

Se demostró la acción antinociceptiva aguda de la amitriptilina cuando es administrada en rangos de 30-50 mg/Kg. Se observó, concomitantemente, como principales manifestaciones neuroconductuales: sedación, piloerección, incoordinación motora, salto y respiración (aumento-disminución) y marcha anormales. 


\section{AGRADECIMIENTOS}

Al doctor Frank Lizarazo Caparó, decano de la Facultad de Medicina Humana de la Universidad de San Martín de Porres, y al doctor Benjamín Castañeda Castañeda, director del Instituto de Investigación.

\section{REFERENCIAS BIBLIOGRÁFICAS}

I. Pérez S, Collado P, González H, Conejo N, Parra A. Estudio de la intervención del sistema colinérgico en el efecto producido por la amitriptilina en una tarea de evitación inhibitoria en ratones machos y hembras [Tesis doctoral]. Valencia, España: Departamento de Psicobiología, Universitat de Valencia; 2007.

2. Bertram G. Katzung, Susan B. Masters, Anthony J. Trevor. Farmacología básica y clínica. 12. ${ }^{a}$ ed. Barcelona, España: McGraw Hill; 20I3. p. 529-37.

3. Stahl SM. Depresión y trastorno bipolar: la psicofarmacología esencial de Stahl. Capítulo 3: Antidepresivos. 3. ${ }^{a}$ ed. Barcelona, España:Aula Médica; 20I0. p. I65-7I.

4. Verdu B, Decosterd I, Buclin T, Stiefel F, Berney A.Antidepressants for the treatment of chronic pain. Drugs. 2008;68(I8):26I I-32.

5. García P. Fisiopatología del dolor. Rev R Acad Cienc Exact Fís Nat. 1999;85(I):83-I5I.

6. Moore RA, Derry S,Aldington D, Cole P,Wiffen PJ.Amitriptyline for neuropathic pain and fibromyalgia in adults. Cochrane Database Syst Rev. 20 12; 12:CD008242.

7. Valverde-Espinoza JA. Recomendaciones de tratamiento farmacológico del dolor neuropático. Neuroeje 20 12;25(2):5I-6I.

8. Vidal MA, Martínez-Fernández E, Martínez-Vázquez de Castro J, Torres LM. Neuropatía diabética. Eficacia de la amitriptilina y de la gabapentina. Rev Soc Esp Dolor. 2004; I I (8):490-504.

9. Cánovas L, Martínez-Salgado J, Barros C, Gómez-Pombo A, Castro-Bande M, Castro-Méndez A. Tratamiento del dolor neuropático: estudio preliminar de amitriptilina frente a nefazodona. Rev Soc Esp Dolor. 2000;7(7):425-30.

10. Calixto-Campos C, Zarpelon AC, Corrêa M, Cardoso RD, Pinho-Ribeiro FA, Cecchini R, et al. The Ehrlich tumor induces pain-like behavior in mice: a novel model of cancer pain for pathophysiological studies and pharmacological screening. Biomed Res Int. 2013;2013:6248I5.

II. Foley KM. Evaluación de la escalera analgésica de la OMS en su 20. ${ }^{\circ}$ aniversario. Rev. Cancer Pain Rel. 2006; I9(I): I-8.

12. Ordóñez-Gallego A, de Castro-Carpeño J. Analgésicos coadyuvantes. En: González-Barón M, Ordóñez-Gallego A, editores. Dolor y cáncer. Hacia una oncología sin dolor. Madrid, España: Panamericana; 2003. p. 261-80.

13. Benítez-del Rosario MA, Pérez-Suárez MC, Fernández-Días R, Cabrejas-Sánchez A. Diagnóstico y tratamiento del dolor oncológico crónico (I).Aten Primaria. 2002;29(6):374-7.

14. Micó JA, Ortega-Álvaro A. Modelos animales del dolor. Reumatol Clin. 2006;2(Supl I): S2-S4.

15. Cusack B, Nelson A, Richelson E. Binding of antidepressants to human brain receptors: focus on newer generation compounds. Psychopharmacology (Berl). 1994; I I4(4):559-65.

16. Lima-Mompó G, Aldana-Vilas L, Casanova-Sotolongo P, Casanova-Carrillo P, Casanova-Carrillo C. Inducción y medición del dolor experimental. Rev Cubana Med Milit. 2003;32(I):49-56.

17. Bannon AW. Models of pain: hot-plate and formalin test in rodents. Curr Protoc Pharmacol. 200I;Chapter 5:Unit 5.7.
18. Roux S, Sablé E, Porsolt RD. Primary observation (Irwin) test in rodents for assessing acute toxicity of a test agent and its effects on behavior and physiological function. Curr Protoc Pharmacol. 2005; Chapter 10:Unit 10.10.

19. Buznego-Rodríguez MT, Fernández-Pérez MD, Llanio-Villate M León-Alonso N, Acevedo-González ME, Pérez-Saad H. Perfil neurofarmacológico del Plectranthus amboinicus (Lour.) Spreng (orégano francés). Potenciación de las esteriotipias inducidas por anfetamina. Rev Cubana Plant Med. 1999;4(I):15-7.

20. Calva-Mercado J], Estudios clínicos experimentales. Salud Pública Méx. 2000;42(4):349-58.

21 . Seuc A.Aleatorización para proteger contra el sesgo de selección en los estudios clínicos sobre asistencia sanitaria: Comentario de la Biblioteca de Salud Reproductiva de la OMS [Internet]. Ginebra, Suiza: Organización Mundial de la Salud; 2012 [citado 28 oct 2014]. Disponible en: http://apps.who.int/rhl/education/ MR0000I2 seuca_com/es/index.html\#.

22. Lazcano-Ponce E, Salazar-Martínez E, Gutiérrez-Castrellón P, Angeles-Llerenas A, Hernández-Garduño A, Viramontes JL. Ensayos clínicos aleatorizados: variantes, métodos de aleatorización, análisis, consideraciones éticas y regulación. Salud Pública Mex. 2004:46(6):559-84.

23. Mujica J, Pulliti Y. Procesamiento de señales in vivo, edición audiovisual y creación de un prototipo de software educativo para simulación de experimentos en farmacología [Tesis para optar el grado de Bachiller en Ingeniería Electrónica]. Lima, Perú: Universidad San Martín de Porres; 2009.

24. van Wilgenburg H. Microlabs for Pharmacologists [Computer based courses in Pharmacology]. Amsterdam, The Netherlands: University of Amsterdam; 2014 [citado 4 nov 2014]. Disponible en: http://oslovet.norecopa.no/produkt.aspx?produkt=538I.

25. Ministerio de Salud del Perú, Instituto Nacional de Salud. Resolución Jefatural I34-2010-J-OPE/INS Conformación del Comité Institucional de Ética para el Uso de Animales en Investigación, y su Modificatoria RJ I88-20II-J-OPE/INS. Lima, Perú: Instituto Nacional de Salud; 201 I.

26. Ministerio de Salud del Perú, Instituto Nacional de Salud. Resolución Jefatural 309-2008-J-OPE/INS Guía de Manejo y Cuidado de Animales de Laboratorio: Ratón. Lima, Perú: Instituto Nacional de Salud; 2008.

27. Paudel KR, Das BP, Rauniar GP, Sangraula H, Deo S, Bhattacharya SK. Antinociceptive effect of amitriptyline in mice of acute pain models. Indian J Exp Biol. 2007;45(6):529-3I.

28. Chogtu B, Bairy KL, Satyam SM, Pirasanthan R, Gupta S.Analgesic modulation of tramadol, amitriptyline and gabapentin in male and female Wistar rats. RJPBCS. 20I3;4(3):70-8.

29. Gray AM, Spencer PS, Sewell RD. The involvement of the opioidergic system in the antinociceptive mechanism of action of antidepressant compounds. Br J Pharmacol. 1998; I24(4):66974.

30. Armijo JA, De las Cuevas I, Adín J. Canales iónicos y epilepsia. Rev Neurol. 2000;30(Supl I):S25-S4I.

31. Cánovas L, Martínez-Salgado J, Barros C, Gómez-Pombo A, Castro-Bande M, Castro-Méndez A. Tratamiento del dolor neuropático: estudio preliminar de amitriptilina frente a nefazodona. Rev Soc Esp Dolor. 2000;7(7):425-30.

32. Fais RS, Reis GM, Dias QM, Rossaneis AC, Silveira JW, Prado WA. Amitriptyline prolongs the antihyperalgesic effect of $2 \mathrm{~Hz}$ electroacupuncture in mononeuropathic rats. Acupunct Rel Ther. 20I3;I (2-3):20-6.

33. Lorenzo P, Moreno A, Lizasoain L, Leza JC, Moro MA, Portolés A. Fármacos antidepresivos y antimaníacos. En: Velásquez. Farmacología básica y clínica. $18^{\mathrm{a}}$ ed. Madrid, España: Panamericana; 2009. p. 309-II. 


\section{Acta Méílica Peruana}

34. Bertram G. Katzung, Susan B. Masters, Anthony J. Trevor. Farmacología básica y clínica. $12^{\mathrm{a}}$ ed. Barcelona, España: McGraw Hill; 2013. p. 530-2.

35. Mobarakeh Jl, Sakurada S, Katsuyama S, Kutsuwa M, Kuramasu $A$, Lin $Z Y$, et al. Role of histamine $H(I)$ receptor in pain perception: a study of the receptor gene knockout mice. Eur J Pharmacol. 2000;39|(I-2):8I-9.

36. Krystal AD, Richelson E, Roth $T$. Review of the histamine system and the clinical effects of $\mathrm{HI}$ antagonists: basis for a new model for understanding the effects of insomnia medications. Sleep Med Rev. 2013;17(4):263-72.
37. Vidal Vademecum Spain. Amitriptilina [Internet]. Madrid, España: Vidal Group; [actualizado 2015, [citado 6 nov 2014]. Disponible en: http://www.vademecum.es/principios-activos-amitriptilinan06aa09.

38. Heerlein A. Tratamientos farmacológicos antidepresivos. Rev Chil Neuro-Psiquiatr. 2002;40(Suppl I):2I-45.

39. Navarro $X$. Fisiología del sistema nervioso autónomo. Rev Neurol. 2002;35(6):553-62

40. Sánchez-López A, Centurión D, Lozano-Cuenca J, Muñoz-Islas E, Cobos-Puc LE, Villalón CM. Receptores de la serotonina que inhiben el tono simpático vasopresor en la rata descerebrada y desmedulada. Arch Cardiol Mex. 2009;79(Supl 2):83-94.

\section{Correspondencia}

Dr. Alberto Salazar-Granara

alberto.salazar@gmail.com

Fecha de recepción: 6 de noviembre de 2014

Fecha de aceptación: 25 de mayo de 2015

\section{Conflictos de interés}

Los autores declaran no tener conflictos de interés durante el planteamiento, ejecución de la investigación y la elaboración del artículo para su publicación 